Cocaine abuse and addiction continue to plague our Nation. Today, about one in six Americans (15 percent in 2007) has tried cocaine by the age of 30 , and 7 percent have tried it by their senior year of high school. But recent discoveries about the inner workings of the brain and the damaging effects of cocaine offer us unprecedented opportunities for addressing this persistent public health problem.

Genetic studies are providing critical information about the hereditary influences on the risk of addiction to psychoactive substances, including cocaine. Moreover, sophisticated imaging technologies have allowed scientists to visualize the brain changes that result from chronic drug exposure or that happen when an addicted person is exposed to drug-associated "cues" that can trigger craving and lead to relapse. By mapping the genetic factors and brain regions responsible for the multiple effects of cocaine, these new technologies can help us identify new targets for treating cocaine addiction.

NIDA remains vigilant in our quest for more effective strategies to address the serious public health issues linked to cocaine abuse. We not only support a wide range of basic and clinical research, but also facilitate the translation of these research findings into real-world settings. To this end, we strive to keep the public informed of the latest scientific advances in the field of addiction. We hope that this compilation of scientific information on cocaine abuse will inform readers and bolster our efforts to tackle the personal and social devastation caused by drug abuse and addiction.

Nora D. Volkow, M.D.

Director

National Institute on Drug Abuse

\section{Research Report Series}
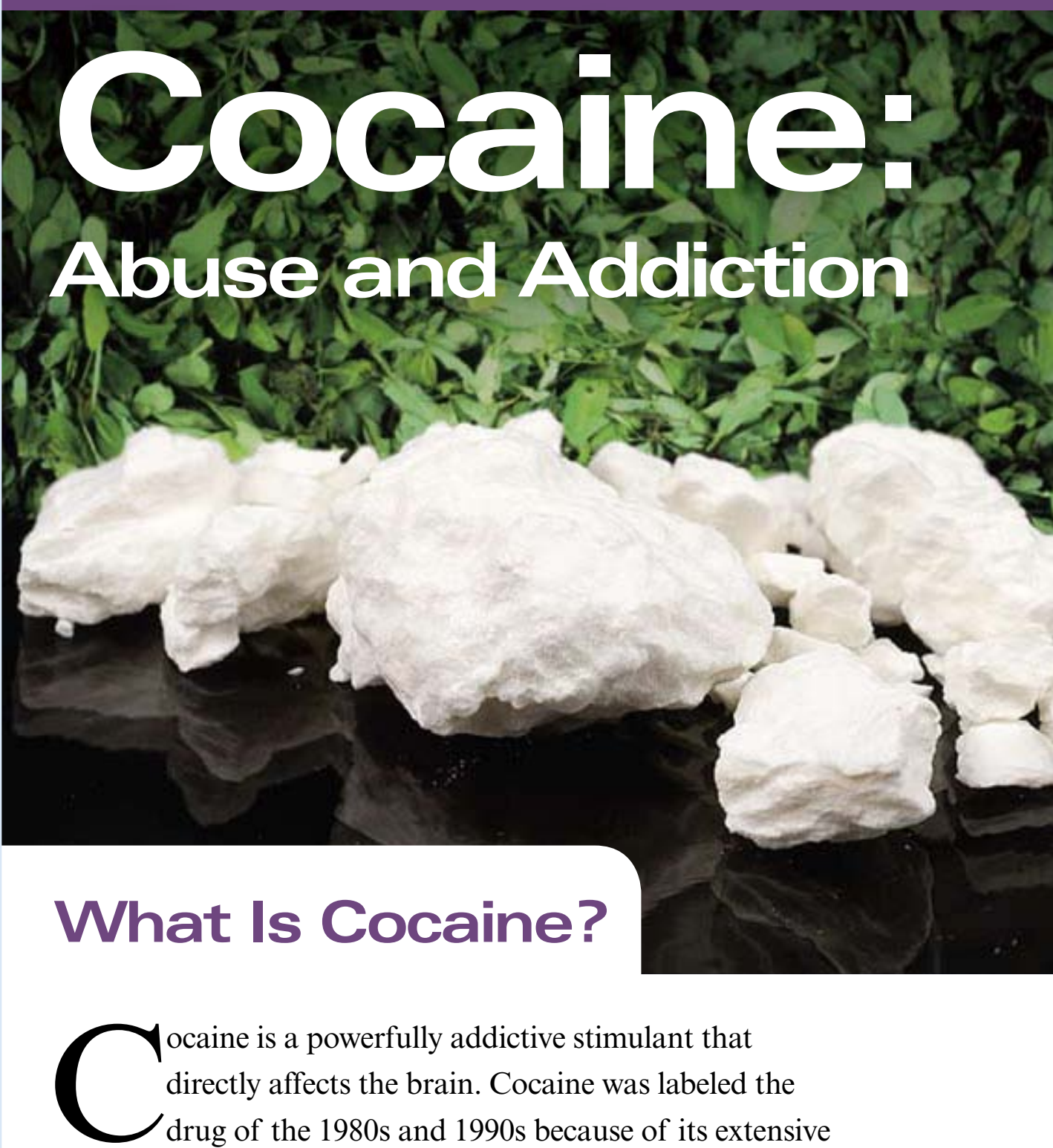

ocaine is a powerfully addictive stimulant that directly affects the brain. Cocaine was labeled the drug of the 1980s and 1990s because of its extensive popularity and use during that period. However, cocaine is not a new drug. In fact, it is one of the oldest known psychoactive substances. Coca leaves, the source of cocaine, have been chewed and ingested for thousands of years, and the purified chemical, cocaine hydrochloride, has been an abused substance for more than 100 years. In the early 1900s, for example, purified cocaine was the main active ingredient in most of the tonics and elixirs 


\section{Research Report Series}

that were developed to treat a wide variety of illnesses.

Pure cocaine was originally extracted from the leaf of the Erythroxylon coca bush, which grew primarily in Peru and Bolivia. After the 1990s, and following crop reduction efforts in those countries, Colombia became the nation with the largest cultivated coca crop. Today, cocaine is a Schedule II drug, which means that it has high potential for abuse but can be administered by a doctor for legitimate medical uses, such as local anesthesia for some eye, ear, and throat surgeries.

Cocaine is generally sold on the street as a fine, white, crystalline powder and is also known as "coke," "C," "snow," "flake," or "blow."

Street dealers generally dilute it with inert substances such as cornstarch, talcum powder, or sugar, or with

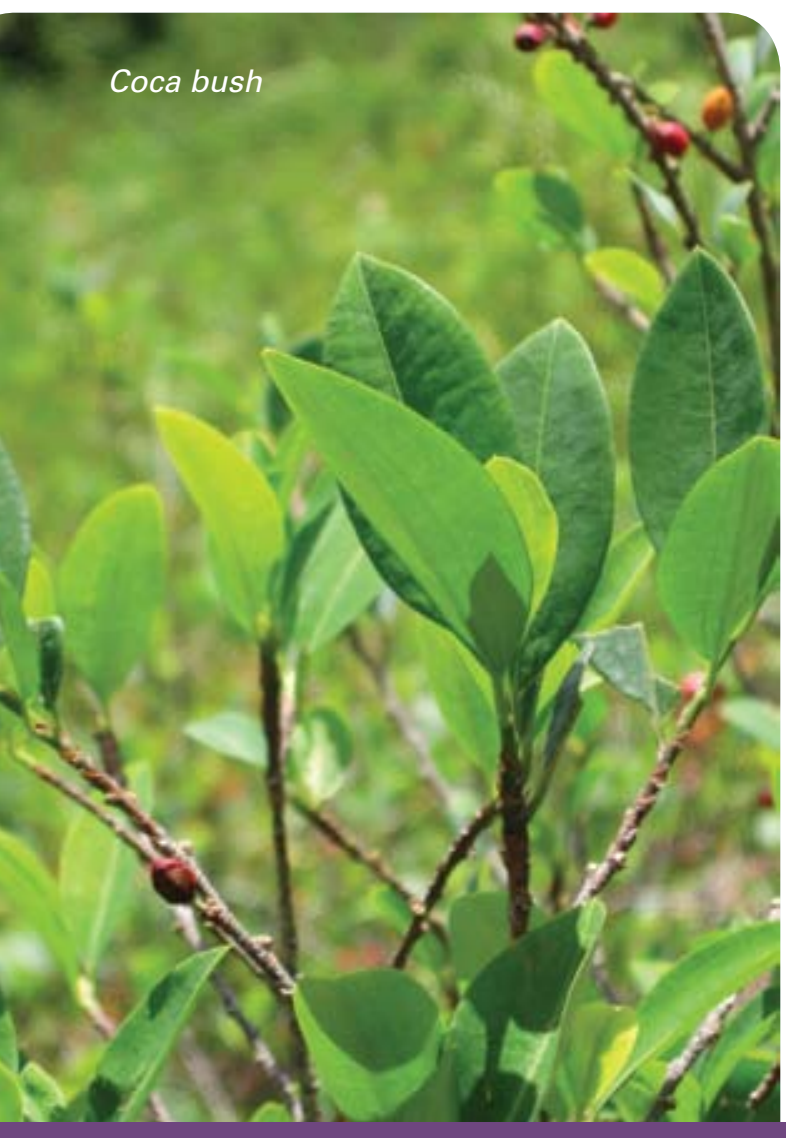

active drugs such as procaine (a chemically related local anesthetic) or amphetamine (another stimulant). Some users combine cocaine with heroin - in what is termed a "speedball."

There are two chemical forms of cocaine that are abused: the water-soluble hydrochloride salt and the water-insoluble cocaine base (or freebase). When abused, the hydrochloride salt, or powdered form of cocaine, can be injected or snorted. The base form of cocaine has been processed with ammonia or sodium bicarbonate (baking soda) and water, and then heated to remove the hydrochloride to produce a smokable substance. The term "crack," which is the street name given to freebase cocaine, refers to the crackling sound heard when the mixture is smoked.

\section{How Is Cocaine Abused?}

The principal routes of cocaine administration are oral, intranasal, intravenous, and inhalation. Snorting, or intranasal administration, is the process of inhaling cocaine powder through the nostrils, where it is absorbed into the bloodstream through the nasal tissues. The drug also can be rubbed onto mucous tissues. Injecting, or intravenous use, releases the drug directly into the bloodstream and heightens the intensity of its effects. Smoking involves inhaling cocaine vapor or smoke into the lungs, where absorption into the bloodstream is as rapid as by injection. This rather immediate and euphoric effect is one of the reasons that crack became enormously popular in the mid-1980s.

Cocaine use ranges from occasional to repeated or compulsive use, with a variety of patterns between these extremes. Other than medical uses, there is no safe way to use cocaine. Any route of administration can lead to absorption of toxic amounts of cocaine, possible acute cardiovascular or cerebrovascular emergencies, and seizures - all of which can result in sudden death.

\section{How Does Cocaine Produce Its Effects?}

Research has led to a clear understanding of how cocaine produces its pleasurable effects and why it is so addictive. Scientists have discovered regions within the brain that are stimulated by all types of reinforcing stimuli such as food, sex, and many drugs of abuse. One neural system that appears to be most affected by cocaine originates in a region of the midbrain called the ventral tegmental area (VTA). Nerve fibers originating in the VTA extend to a region known as the nucleus accumbens, one of the brain's key areas involved in reward. Animal studies show that rewards increase levels of the brain chemical (or neurotransmitter) dopamine, thereby increasing neural activity in the nucleus accumbens. In the normal communication process, dopamine is released by a neuron into the synapse (the small gap between two neurons), where it binds to specialized proteins (called dopamine receptors) on the neighboring neuron and sends a signal 

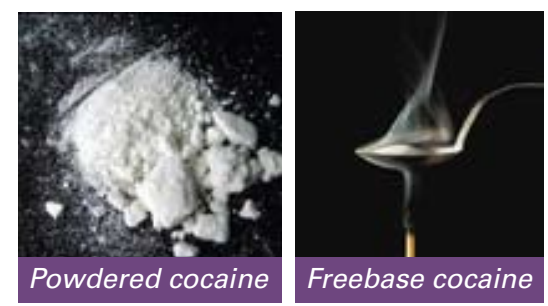

to that neuron. Dopamine is then removed from the synapse to be recycled for further use. Drugs of abuse can interfere with this normal communication process. For example, scientists have discovered that cocaine acts by blocking the removal of dopamine from the synapse, which results in an accumulation of dopamine and an amplified signal to the receiving neurons (see image on page 4, "Cocaine in the brain"). This is what causes the initial euphoria commonly reported by cocaine abusers.

\section{What Are the Short-Term Effects of Cocaine Use?}

Cocaine's effects appear almost immediately after a single dose and disappear within a few minutes or within an hour. Taken in small amounts, cocaine usually makes the user feel euphoric, energetic, talkative, and mentally alert, especially to the sensations of sight, sound, and touch. It can also temporarily decrease the need for food and sleep. Some users find that the drug helps them perform simple physical and intellectual tasks more quickly, although others experience the opposite effect.

The duration of cocaine's euphoric effect depends upon the route of administration. The

\section{What Is the Scope of Cocaine Use in the United States?}

The National Survey on Drug Use and Health (NSDUH) estimates that in 2007 there were 2.1 million current (past-month) cocaine users, of which approximately 610,000 were current crack users. Adults aged 18 to 25 years have a higher rate of current cocaine use than any other age group, with 1.7 percent of young adults reporting past month cocaine use. Overall, men report higher rates of current cocaine use than women. Ethnic/ racial differences also occur-with the highest rates in those reporting two or more races (1.1 percent), followed by Hispanics (1.0 percent), Whites (0.9 percent), and AfricanAmericans (0.8 percent).

The 2008 Monitoring the Future survey, which annually surveys teen attitudes and drug use, reports that while there has been a significant decline in the 30-day prevalence of powder cocaine use among 8th-, 10th-, and 12th-graders from its peak use in the late 1990s, there was no significant change in current cocaine use from 2001 to 2008; however, crack use declined signifi-

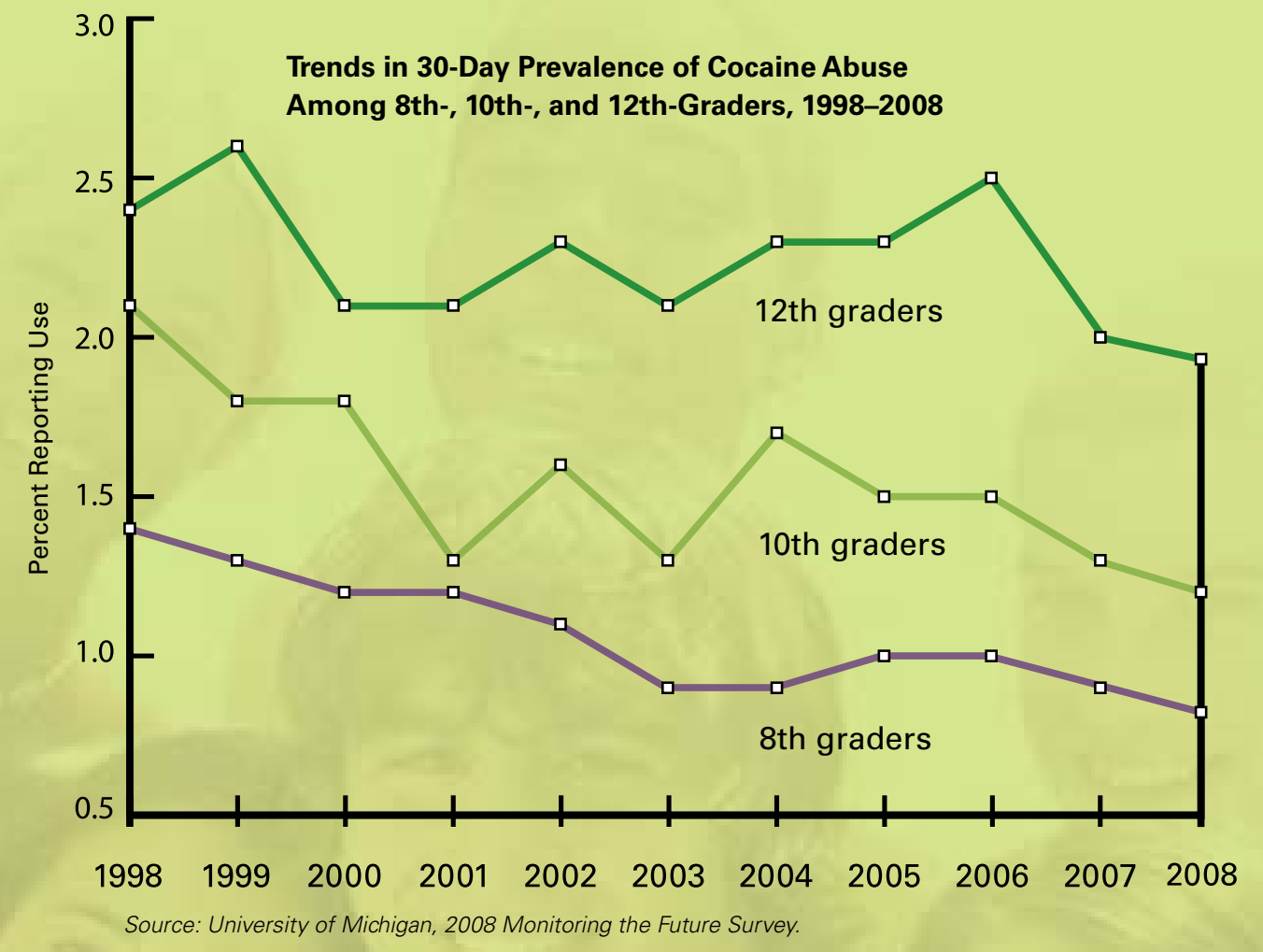

cantly during this timeframe among 8th-and 12th-graders.

Repeated cocaine use can produce addiction and other adverse health consequences. In 2007, according to the NSDUH, nearly 1.6 million Americans met Diagnostic and Statistical Manual of Mental Disorders criteria for dependence or abuse of cocaine (in any form) in the past
12 months. Further, data from the 2005 Drug Abuse Warning Network (DAWN) report showed that cocaine was involved in 448,481 of the total 1,449,154 visits to emergency departments for drug misuse or abuse. This translates to almost one in three drug misuse or abuse emergency department visits (31 percent) that involved cocaine. 
faster the drug is absorbed, the more intense the resulting high, but also the shorter the duration. The high from snorting is relatively slow to arrive but may last 15 to 30 minutes; in contrast, the effects from smoking are more immediate but may last only 5 to 10 minutes.

The short-term physiological effects of cocaine include constricted blood vessels; dilated pupils; and increased temperature, heart rate, and blood pressure. Large amounts of cocaine may intensify the user's high but can also lead to bizarre, erratic, and violent behavior. Some cocaine users report feelings of restlessness, irritability, and anxiousness. Users may also experience tremors, vertigo, muscle twitches, or paranoia. There can also be severe medical complications associated with cocaine abuse. Some of the most frequent are cardiovascular effects, including disturbances in heart rhythm and heart attacks; neurological effects, including strokes, seizures, headaches, and even coma; and gastrointestinal complications, including abdominal pain and nausea. In rare instances, sudden death can occur on the first use of cocaine or unexpectedly thereafter. Cocainerelated deaths are often a result of cardiac arrest or seizures followed by respiratory arrest.

Research has also revealed a potentially dangerous interaction between cocaine and alcohol. In fact, this mixture is the most common two-drug combination that results in drug-related death.

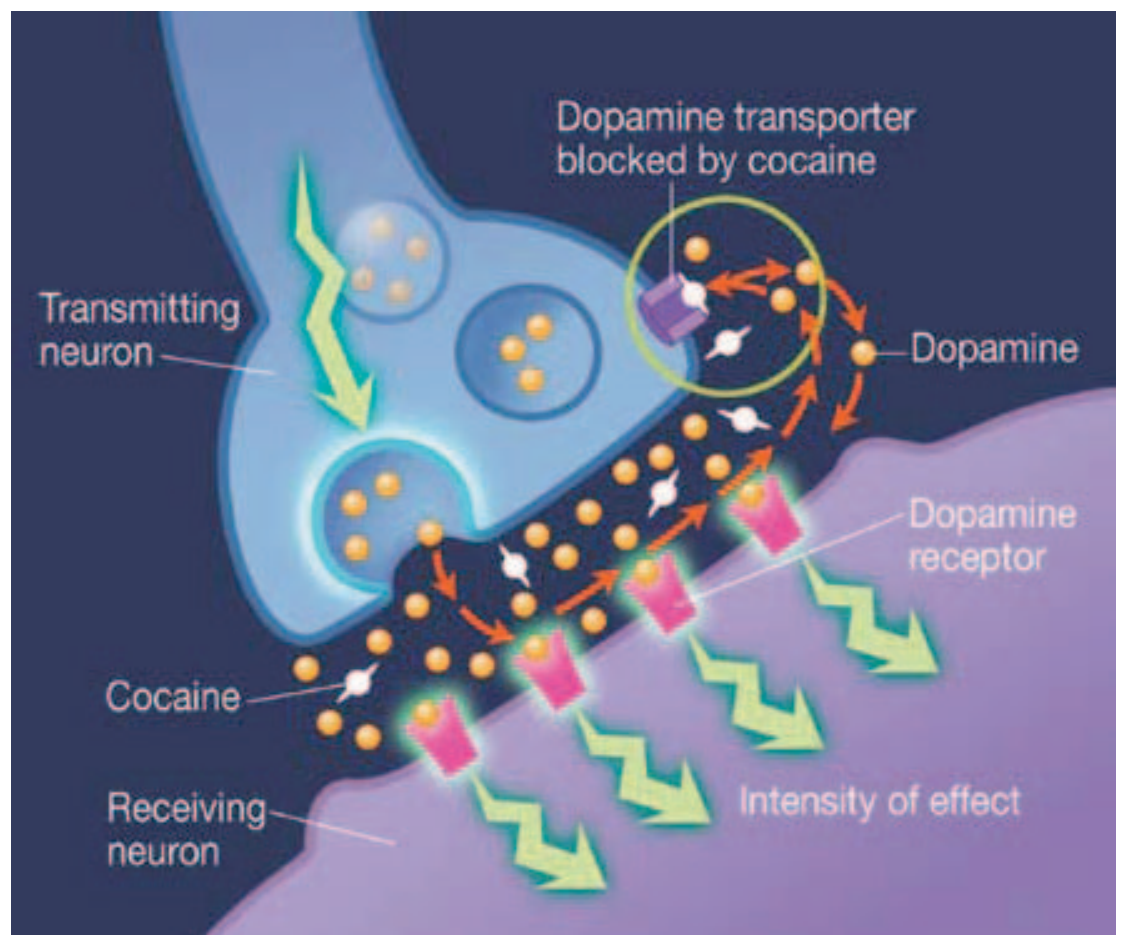

Cocaine in the brain - In the normal communication process, dopamine is released by a neuron into the synapse, where it can bind to dopamine receptors on neighboring neurons. Normally, dopamine is then recycled back into the transmitting neuron by a specialized protein called the dopamine transporter. If cocaine is present, it attaches to the dopamine transporter and blocks the normal recycling process, resulting in a buildup of dopamine in the synapse, which contributes to the pleasurable effects of cocaine.

\section{What Are the Long-Term Effects of Cocaine Use?}

Cocaine is a powerfully addictive drug. Thus, it is unlikely that an individual will be able to reliably predict or control the extent to which he or she will continue to want or use the drug. And, if addiction takes hold, the risk for relapse is high even following long periods of abstinence. Recent studies have shown that during periods of abstinence, the memory of the cocaine experience or exposure to cues associated with drug use can trigger tremendous craving and relapse to drug use.

With repeated exposure to cocaine, the brain starts to adapt, and the reward pathway becomes less sensitive to natural reinforcers and to the drug itself. Tolerance may develop - this means that higher doses and/or more frequent use of cocaine is needed to register the same level of pleasure experienced during initial use. At the same time, users can also become more sensitive (sensitization) to cocaine's anxiety-producing, convulsant, and other toxic effects.

Users take cocaine in "binges," during which the cocaine is used repeatedly and at increasingly higher doses. This can lead to increased irritability, restlessness, and paranoia - even a full-blown paranoid psychosis, in which the individual loses touch with reality and experiences auditory hallucinations. With increasing dosages or frequency of use, the risk of adverse psychological or physiological effects increases.

Different routes of cocaine administration can produce different adverse effects. Regularly snorting cocaine, for example, can lead to loss of sense of smell; nosebleeds; problems with swal- 


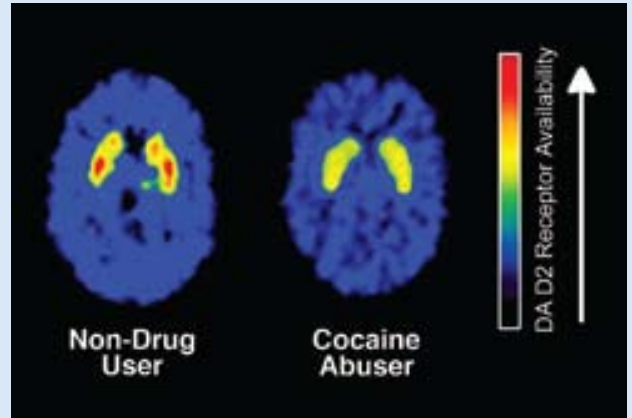

lowing; hoarseness; and an overall irritation of the nasal septum, which could result in a chronically inflamed, runny nose. Ingested cocaine can cause severe bowel gangrene, due to reduced blood flow. Persons who inject cocaine have puncture marks called "tracks," most commonly in their forearms, and may experience allergic reactions, either to the drug or to some additive in street cocaine, which in severe cases can result in death. Many chronic cocaine users lose their appetite and experience significant weight loss and malnourishment.

\section{Are Cocaine Abusers at Risk for Contracting HIVIAIDS and Hepatitis?}

Yes, cocaine abusers are at increased risk for contracting such infectious diseases as human immunodeficiency virus/acquired immune deficiency syndrome (HIV/AIDS) and viral hepatitis. This risk stems not only from sharing contaminated needles and drug paraphernalia but also from engaging in risky behaviors as a result of intoxication. Research has shown that drug intoxication and addiction can compromise
Brain images showing decreased dopamine $\left(D_{2}\right)$ receptors in the brain of a person addicted to cocaine versus a non-drug user. The dopamine system is important for conditioning and motivation, and alterations such as this are likely responsible, in part, for the diminished sensitivity to natural rewards that develops with addiction. percent in 2006) who seek treatment for cocaine abuse smoke crack and are likely to be polydrug abusers, or users of more than one substance. The widespread abuse of cocaine has stimulated extensive efforts to develop treatment programs for cocaine. As with any drug addiction, this is a complex disease that involves biological changes in the brain as well as myriad social, familial, and other environmental problems. Therefore, treatment of cocaine addiction must be comprehensive, and strategies need to assess the neurobiological, social, and medical aspects of the patient's drug abuse. Moreover, patients who have a variety of addictions often have other co-occurring mental disorders that require additional behavioral or pharmacological interventions.

\section{Pharmacological Approaches}

Presently, there are no FDAapproved medications to treat cocaine addiction. Consequently, NIDA is aggressively working to identify and test new medications to treat cocaine addiction safely and effectively. Several medications marketed for other diseases (e.g., baclofen, modafinil, tiagabine, disulfiram, and topiramate) show promise and have been reported to reduce cocaine use in controlled clinical trials. Among these, disulfiram (used to treat alcoholism) has produced the most consistent reductions in cocaine abuse. On the other hand, new knowledge of how the brain is changed by cocaine is directing attention to novel targets for medications development. Compounds that are currently being tested for addiction treatment take advantage of underlying cocaine-induced adaptations in the brain that disturb the balance between excitatory (glutamate) and inhibitory (gammaaminobutyric acid) neurotransmission. Also, dopamine D3 receptors
In 2006, cocaine accounted for about 14 percent of all admissions to drug abuse treatment programs. The majority of individuals (71

\section{What Treatments Are Effective for Cocaine Abusers?}

judgment and decisionmaking,
and potentially lead to risky sexu encounters, needle sharing, and trading sex for drugs - by both men
and women. In fact, some studies are showing that among drug abusers, those who do not inject drugs
are contracting HIV at rates equal to tho who do inject drugs, further mission of HIV in this population. has spread rapidly among injecting drug users. Nearly 50 percent are exposed within 2 years of initiating rates are between 40 and 98 percent in those injecting for more than 2 years. Although treatment for $\mathrm{HCV}$ have significant side effects, medical followup is essential for all those cine for the hepatitis $\mathrm{C}$ virus, and it ghly transmissible via injection for any individual who has ever injected drugs. 


\section{What Are the Effects of Maternal Cocaine Use?}

The full extent of the effects of prenatal cocaine exposure on a child are not completely known, but many scientific studies have documented that babies born to mothers who abuse cocaine during pregnancy are often prematurely delivered, have low birthweights and smaller head circumferences, and are shorter in length than babies born to mothers who do not abuse cocaine.

Nevertheless, it is difficult to estimate the full extent of the consequences of maternal drug abuse and to determine the specific hazard of a particular drug to the unborn child. This is because multiple factors-

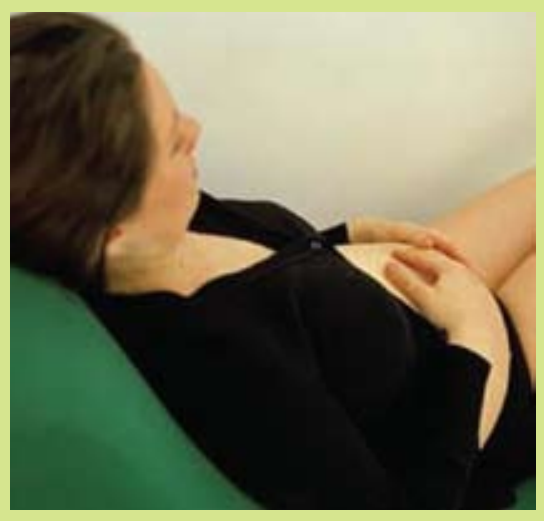

such as the amount and number of all drugs abused, including nicotine; extent of prenatal care; possible neglect or abuse of the child; exposure to violence in the environment; socioeconomic conditions; maternal nutrition; other health conditions; and exposure to sexually transmitted diseases-can all interact to impact maternal, fetal, and child outcomes.
Some may recall that "crack babies," or babies born to mothers who abused crack cocaine while pregnant, were at one time written off as a lost generation. They were predicted to suffer from severe, irreversible damage, including reduced intelligence and social skills. It was later found that this was a gross exaggeration. However, the fact that most of these children appear normal should not be overinterpreted to indicate that there is no cause for concern. Using sophisticated technologies, scientists are now finding that exposure to cocaine during fetal development may lead to subtle, yet significant, later deficits in some children, including deficits in some aspects of cognitive performance, information processing, and attention to tasks-abilities that are important for the realization of a child's full potential. (a subtype of dopamine receptor) constitute a novel molecular target of high interest. Medications that act at these receptors are just now being tested for safety in humans. Finally, a cocaine vaccine that prevents entry of cocaine into the brain holds great promise for reducing the risk of relapse. In addition to treatments for addiction, medical treatments are being developed to address the acute emergencies that result from cocaine overdose each year.

\section{Behavioral Interventions}

Many behavioral treatments for cocaine addiction have proven to be effective in both residential and outpatient settings. Indeed, behavioral therapies are often the only available and effective treatments for many drug problems, including stimulant addictions. However, the integration of behavioral and pharmacological treatments may ultimately prove to be the most effective approach.

One form of behavioral therapy that is showing positive results in

\section{Presently, there \\ are no proven \\ medications to treat \\ cocaine addiction. \\ Consequently, NIDA \\ is aggressively \\ working to identify \\ and test new \\ medications.}

cocaine-addicted populations is contingency management, or motivational incentives (MI). MI may be particularly useful for helping patients achieve initial abstinence from cocaine and for helping patients stay in treatment. Programs use a voucher or prize-based system that rewards patients who abstain from cocaine and other drug use. On the basis of drug-free urine tests, the patients earn points, or chips, which can be exchanged for items that encourage healthy living, such as joining a gym or going to a movie or dinner. This approach has recently been shown to be practical and effective in community treatment programs.

Cognitive-behavioral therapy (CBT) is an effective approach for preventing relapse. CBT is focused on helping cocaine-addicted individuals abstain — and remain abstinent_-from cocaine and other substances. The underlying assumption is that learning processes play an important role in the development and continuation of cocaine abuse and addiction. These same learning processes can be harnessed to help individuals reduce drug use and successfully prevent relapse. This approach attempts to help patients recognize, avoid, and cope; that is, they recognize the situations in which they are most likely to use cocaine, avoid these situations when appropriate, and cope more effectively with a range of problems and problematic behaviors associated with drug abuse. This therapy is also noteworthy because of its compatibility with a range of other treatments patients may receive, including pharmacotherapy. 
Therapeutic communities (TCs), or residential programs, offer another alternative to persons in need of treatment for cocaine addiction. TCs usually require a 6- or 12-month stay and use the program's entire "community" as active components of treatment. They can include onsite vocational rehabilitation and other supportive services and focus on successful reinsertion of the individual into society.

Community-based recovery groups - such as Cocaine Anonymous - which use a 12-step program, can also be helpful to people trying to sustain abstinence. Participants may benefit from supportive fellowship and sharing with those experiencing common problems and issues.
It is important that patients receive services that match all of their treatment needs. For example, if a patient is unemployed, it may be helpful to provide vocational rehabilitation or career counseling along with addiction treatment. If a patient has marital problems, it may be important to offer couples counseling.

\section{Glossary}

Addiction: A chronic, relapsing disease characterized by compulsive drug seeking and use and by neurochemical and molecular changes in the brain.

Anesthetic: An agent that causes insensitivity to pain.

Coca: The plant, Erythroxylon, from which cocaine is derived. Also refers to the leaves of this plant.

Cocaethylene: A potent stimulant formed in the body when cocaine and alcohol are used together.

Crack: The slang term for a smokable form of cocaine.

Craving: A powerful, often uncontrollable, desire for drugs.

Dopamine: A neurotransmitter present in regions of the brain that regulate movement, emotion, motivation, and the feeling of pleasure.
Freebase: A solid, waterinsoluble, and smokable form of cocaine that is produced when its hydrochloride salt form is processed with ammonia or sodium bicarbonate, and water, then heated to remove the hydrochloride. (Also, see "crack.")

Frontal cortex: The front part of the brain involved with reasoning, planning, problemsolving, and other higher cognitive functions.

\section{Gamma-aminobutyric acid}

(GABA): The main inhibitory neurotransmitter in the central nervous system. GABA provides the needed counterbalance to the actions of other systems, particularly the excitatory neurotransmitter glutamate.

Glutamate: An excitatory neurotransmitter found throughout the brain, that influences the reward system and is involved in learning and memory among other functions.

Hydrochloride salt: A powdered, water-soluble form of cocaine that can be injected or snorted.

Neuron: A nerve cell.

Nucleus accumbens: $A$ brain region involved in motivation and reward. Nearly all drugs of abuse directly or indirectly increase dopamine in the nucleus accumbens, contributing to their addictive properties.

Physical dependence: A state in which the body adapts to a drug and where withdrawal occurs if use of the drug is stopped abruptly. Physical dependence can happen with chronic-even appropriate-use of many drugs, and in and of itself does not constitute addiction.
Polydrug user: An individual who uses more than one drug.

Rush: A surge of pleasure that rapidly follows administration of some drugs.

Stimulant: A class of drugs that increase or enhance the activity of monamines (such as dopamine) in the brain. Stimulants increase arousal, heart rate, blood pressure, and respiration, and decrease appetite. Includes some medications used to treat attention-deficit hyperactivity disorder (e.g., methylphenidate and amphetamines), as well as cocaine and methamphetamine.

Tolerance: A condition in which higher doses of a drug are required to produce the same effect as during initial use.

Vertigo: The sensation of dizziness.

\section{References}

Alessi, S.M.; Hanson, T.; Wieners, M.; and Petry, N.M. Low-cost contingency management in community clinics: Delivering incentives partially in group therapy. Experimental and Clinical Psychopharmacology 15(3):293-300, 2007.
Des Jarlais, D.C., et al. Convergence of HIV seroprevalence among injecting and non-injecting drug users in New York City. AIDS 21(2):231-235, 2007.

Gold, M.S. Cocaine (and crack): Clinical aspects. In: Lowinson, J.H., ed. Substance Abuse: A Comprehensive Textbook, 3rd edition, Baltimore: Williams \& Wilkins, pp. 181-198, 1997.

Hagan, H., et al. HCV Synthesis Project: Preliminary analyses of $\mathrm{HCV}$ prevalence in relation to age and duration of injection. International Journal of Drug Policy 18:341-351, 2007.
Institute for Social Research Monitoring the Future, 2008 (Study Results). Ann Arbor, MI: University of Michigan, 2008. Data retrieved 12/11/2008 from http://www. monitoringthefuture.org. 


\section{References}

continued from page 7

Martell, B.A., et al. Vaccine pharmacotherapy for the treatment of cocaine dependence. Biological Psychiatry 58(2):158-164, 2005.

Shoptaw, S., et al. Randomized placebo-controlled trial of baclofen for cocaine dependence: Preliminary effects for individuals with chronic patterns of cocaine use. Journal of Clinical Psychiatry 64(12):1440-1448, 2003.
Snyder, S.H. Drugs and the Brain. New York: Scientific American Library, 1996.

Sokoloff, P., et al. The dopamine D3 receptor: A therapeutic target for the treatment of neuropsychiatric disorders. CNS and Neurological Disorders-Drug Targets 5(1):2543, 2006.

Substance Abuse and Mental Health Services Administration,
Office of Applied Studies. Results from the 2007 National Survey on Drug Use and Health: National Findings. DHHS Pub. No. SMA 08-4343, Rockville, MD: SAMHSA, 2008.

Suh, J.J.; Pettinati, H.M.; Kampman, K.M.; and O'Brien, C.P. The status of disulfiram: A half of a century later. Journal of Clinical Psychopharmacology 26(3):290-302, 2006.
Tseng, F.-C., et al. Seroprevalence of hepatitis $C$ virus and hepatitis B virus among San Francisco injection drug users, 1998 to 2000. Hepatology 46(3):666-671, 2007.

Volkow, N.D., et al. Decreased striatal dopaminergic responsiveness in detoxified cocainedependent subjects. Nature 386(6627):830-833, 1997.

\section{Where Can I Get More Scientific Information About Cocaine Abuse and Addiction?}

To learn more about cocaine and other drugs of abuse, or to order materials on these topics free of charge in English or Spanish, visit the NIDA Web site at www.drugabuse.gov凹 or contact the DrugPubs Research Dissemination Center at 877-NIDA-NIH (877-643-2644; TTY/TDD: 240-645-0228).

\section{won DRUG PUBS RESEARCH DISSEMINATION CENTER}

What's New on the NIDA Web Site

- Information on drugs of abuse

- Publications and communications (including NIDA Notes and Addiction Science \& Clinical Practice journal)

- Calendar of events

- Links to NIDA organizational units

- Funding information (including program announcements and deadlines)

- International activities

- Links to related Web sites (access to Web sites of many other organizations in the field)
NIDA Web Sites

drugabuse.gov

backtoschool.drugabuse.gov

teens.drugabuse.gov

For Physician Information

\section{NIDAMED}

www.drugabuse.gov/nidamed

\section{Other Web Sites}

Information on cocaine abuse and addiction is also available through the following Web site:

- Substance Abuse and Mental Health Services Administration Health Information Network: www.samhsa.gov/shin

\section{U.S. Department of Health and Human Services}

NATIONAL INSTITUTES OF HEALTH NIDA

NIH Publication Number 09-4166

Printed May 1999, Revised November 2004,

Revised May 2009

Feel free to reprint this publication. 